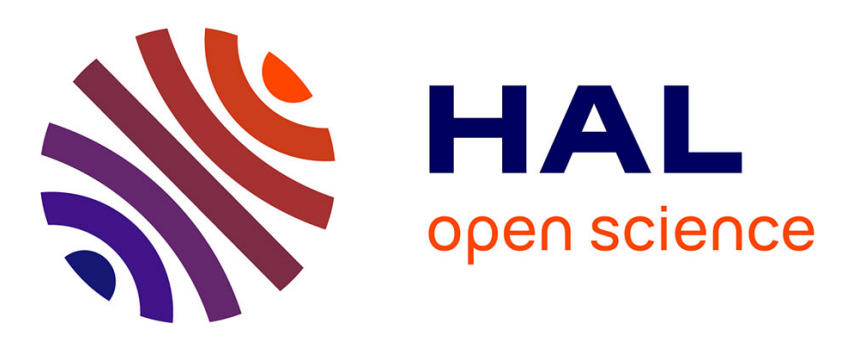

\title{
On the energy transfer to high frequencies in the damped/driven nonlinear Schrödinger equation
}

\author{
Guan Huang, Sergei Kuksin
}

\section{To cite this version:}

Guan Huang, Sergei Kuksin. On the energy transfer to high frequencies in the damped/driven nonlinear Schrödinger equation. Stochastics and Partial Differential Equations: Analysis and Computations, In press, 10.1007/s40072-020-00187-2 . hal-03112246

\section{HAL Id: hal-03112246 \\ https://hal.science/hal-03112246}

Submitted on 16 Jan 2021

HAL is a multi-disciplinary open access archive for the deposit and dissemination of scientific research documents, whether they are published or not. The documents may come from teaching and research institutions in France or abroad, or from public or private research centers.
L'archive ouverte pluridisciplinaire HAL, est destinée au dépôt et à la diffusion de documents scientifiques de niveau recherche, publiés ou non, émanant des établissements d'enseignement et de recherche français ou étrangers, des laboratoires publics ou privés. 


\title{
ON THE ENERGY TRANSFER TO HIGH FREQUENCIES IN THE DAMPED/DRIVEN NONLINEAR SCHRÖDINGER EQUATION
}

\author{
GUAN HUANG AND SERGEI KUKSIN
}

\begin{abstract}
We consider a damped/driven nonlinear Schrödinger equation in $\mathbb{R}^{n}$, where $n$ is arbitrary,

$$
\mathbb{E} u_{t}-\nu \Delta u+i|u|^{2} u=\sqrt{\nu} \eta(t, x), \quad \nu>0,
$$

under odd periodic boundary conditions. Here $\eta(t, x)$ is a random force which is white in time and smooth in space. It is known that the Sobolev norms of solutions satisfy $\|u(t)\|_{m}^{2} \leq C \nu^{-m}$, uniformly in $t \geq 0$ and $\nu>0$. In this work we prove that for small $\nu>0$ and any initial data, with large probability the Sobolev norms $\|u(t, \cdot)\|_{m}$ with $m>2$ become large at least to the order of $\nu^{-\kappa_{n, m}}$ with $\kappa_{n, m}>0$, on time intervals of order $\mathcal{O}\left(\frac{1}{\nu}\right)$. It proves that solutions of the equation develop short space-scale of order $\nu$ to a positive degree, and rigorously establishes the (direct) cascade of energy for the equation.
\end{abstract}

\section{INTRODUCTION}

In this work we study a damped/driven nonlinear Schrödinger equation

$$
u_{t}-\nu \Delta u+i|u|^{2} u=\sqrt{\nu} \eta(t, x), \quad x \in \mathbb{R}^{n},
$$

i.e. a CGL equation without linear dispersion, with cubic Hamiltonian nonlinearity and a random forcing. The dimension $n$ is any, $0<\nu \leq 1$ is the viscosity constant and the random force $\eta$ is white in time $t$ and regular in $x$. The equation is considered under the odd periodic boundary conditions,

$$
u\left(t, \ldots, x_{j}, \ldots\right)=u\left(t, \ldots, x_{j}+2 \pi, \ldots\right)=-u\left(t, \ldots, x_{j}+\pi, \ldots\right), \quad j=1, \ldots, n .
$$

The latter implies that $u$ vanishes on the boundary of the cube of half-periods $K^{n}=[0, \pi]^{n}$,

$$
\left.u\right|_{\partial K^{n}}=0 \text {. }
$$

We denote by $\left\{\varphi_{d}(\cdot), d=\left(d_{1}, \ldots, d_{n}\right) \in \mathbb{N}^{n}\right\}$ the trigonometric basis in the space of odd periodic functions,

$$
\varphi_{d}(x)=\left(\frac{2}{\pi}\right)^{\frac{n}{2}} \sin \left(d_{1} x_{1}\right) \cdots \sin \left(d_{n} x_{n}\right) .
$$

The basis is orthonormal with respect to the normalised scalar product $\langle\langle\cdot, \cdot\rangle$ in $L_{2}\left(K^{n}, \pi^{-n} d x\right)$,

$$
\langle u, v\rangle\rangle=\int_{K^{n}}\langle u(x), v(x)\rangle \pi^{-n} d x,
$$

where $\langle\cdot, \cdot\rangle$ is the real scalar product in $\mathbb{C},\langle u, v\rangle=\mathfrak{R} u \bar{v}$. It is formed by eigenfunctions of the Laplacian:

$$
(-\Delta) \varphi_{d}=|d|^{2} \varphi_{d}
$$

The force $\eta(t, x)$ is a random field of the form

$$
\eta(t, x)=\frac{\partial}{\partial t} \xi(t, x), \quad \xi(t, x)=\sum_{d \in \mathbb{N}^{n}} b_{d} \beta_{d}(t) \varphi_{d}(x) .
$$

Here $\beta_{d}(t)=\beta_{d}^{R}(t)+i \beta_{d}^{I}(t)$, where $\beta_{d}^{R}(t), \beta_{d}^{I}(t)$ are independent real-valued standard Brownian motions, defined on a complete probability space $(\Omega, \mathcal{F}, \mathbb{P})$ with a 
filtration $\left\{\mathcal{F}_{t} ; t \geqslant 0\right\}$. The set of real numbers $\left\{b_{d}, d \in \mathbb{N}^{n}\right\}$ is assumed to form a non-zero sequence, satisfying

$$
0<B_{m_{*}}<\infty, \quad m_{*}=\min \{m \in \mathbb{Z}: m>n / 2\},
$$

where for a real number $k$ we set

$$
B_{k}:=\sum_{d \in \mathbb{N}^{n}}|d|^{2 k}\left|b_{d}\right|^{2} \leq \infty .
$$

For $m \geq 0$ we denote by $H^{m}$ the Sobolev space of order $m$, formed by complex odd periodic functions, equipped with the homogeneous norm,

$$
\|u\|_{m}=\left\|(-\Delta)^{\frac{m}{2}} u\right\|_{0},
$$

where $\|\cdot\|_{0}$ is the $L^{2}$-norm on $K^{n},\|u\|_{0}^{2}=\left\langle\langle u, u\rangle\right.$ (see (1.2)). If we write $u \in H^{m}$ as Fourier series, $u(x)=\sum_{d \in \mathbb{N}^{n}} u_{d} \varphi_{d}(x)$, then $\|u\|_{m}^{2}=\sum_{d \in \mathbb{N}^{n}}|d|^{2 m}\left|u_{d}\right|^{2}$.

Eq. (1.1) with small $\nu$ belongs to a group of equations, describing turbulence in the CGL equations. These equations have got quite a lot of attention in physical literature as models for turbulence in various media, e.g. see [3, Chapter 5]. In particular - as a natural model for hydrodynamical turbulence since eq. (1.1) is obtained from the Navier-Stokes system by replacing the Euler term $(u \cdot \nabla) u$, which is a quadratic Hamiltonian nonlinearity, by $i|u|^{2} u$, which is a cubic Hamiltonian nonlinearity, see [13].

The global solvability of eq. (1.1) for any space dimension $n$ is established in [8, 10]. It is proved there that if

$$
u(0, x)=u_{0}(x),
$$

where $u_{0} \in H^{m} \cap C\left(K^{n}\right), m \in \mathbb{N}$, and if $B_{m}<\infty$, then the problem (1.1), (1.5) has a unique strong solution $u(t, x)$ in $H^{m}$ which we write as $u\left(t, x ; u_{0}\right)$, or $u\left(t ; u_{0}\right)$, or $u_{\nu}\left(t ; u_{0}\right)$. Its norm satisfies

$$
\mathbb{E}\left\|u\left(t ; u_{0}\right)\right\|_{m}^{2} \leq C_{m} \nu^{-m}, \quad t \geq 0,
$$

where $C_{m}$ depends on $\left\|u_{0}\right\|_{m},\left|u_{0}\right|_{\infty}$ and $B_{m}, B_{m_{*}}$. Furthermore, denoting by $C_{0}\left(K^{n}\right)$ the space of continuous complex functions on $K^{n}$, vanishing at $\partial K^{n}$, we have that the solutions $u(t, x)$ define a Markov process in $C_{0}\left(K^{n}\right)$. Moreover, if the noise $\eta(t, \cdot)$ is non-degenerate in the sense that in (1.3) all coefficients $b_{d}$ are non-zero, then this process is mixing. ${ }^{1}$

Our goal is to study the growth of higher Sobolev norms for solutions of eq. (1.1) as $\nu \rightarrow 0$ on time intervals of order $\mathcal{O}\left(\frac{1}{\nu}\right)$. The main result of this work is the following.

Theorem 1. For any real number $m>2$, in addition to (1.4), assume that $B_{m}<\infty$. Then there exists $\kappa_{n, m}>0$ such that for every fixed quadruple $\left(\delta, \kappa, \mathscr{K}, T_{0}\right)$, where

$$
\kappa \in\left(0, \kappa_{n, m}\right), \quad \delta \in\left(0, \frac{1}{8}\right), \quad \mathscr{K}, T_{0}>0,
$$

there exists a $\nu_{0}>0$ with the property that if $0<\nu \leq \nu_{0}$, then for every $u_{0} \epsilon$ $H^{m} \cap C_{0}\left(K^{n}\right)$, satisfying

$$
\left|u_{0}\right|_{\infty} \leqslant \mathscr{K}, \quad\left\|u_{0}\right\|_{m} \leq \nu^{-\kappa m},
$$

the solution $u\left(t, x ; u_{0}\right)$ is such that

(1)

$$
\mathbb{P}\left\{\sup _{t \in\left[t_{0}, t_{0}+T_{0} \nu^{-1}\right]}\left\|u_{\nu}^{\omega}(t)\right\|_{m}>\nu^{-m \kappa}\right\} \geq 1-\delta, \quad \forall t_{0} \geq 0
$$

\footnotetext{
${ }^{1}$ We note that solutions of equations (1.1) with complex $\nu$ behave differently, and solubility of those equations with large $n$ is unknown.
} 
(2) If $m$ is an integer, $m \geq 3$, then a possible choice of $\kappa_{n, m}$ is $\kappa_{n, m}=\frac{1}{35}$, and there exists $C \geq 1$, depending on $\kappa<\frac{1}{35}, \mathscr{K}, m, B_{m_{*}}$ and $B_{m}$, such that

$$
C^{-1} \nu^{-2 m \kappa+1} \leqslant \mathbb{E}\left(\nu \int_{t_{0}}^{t_{0}+\nu^{-1}}\left\|u_{\nu}(s)\right\|_{m}^{2} d s\right) \leqslant C \nu^{-m}, \quad \forall t_{0} \geq 0 .
$$

A similar result holds for the classical $C^{k}$-norms of solutions:

Proposition 2. For any integer $m \geq 2$ in addition to (1.4) assume that $B_{m}<\infty$. Then for every fixed triplet $K, \mathcal{K}, T_{0}>0$ and any $0<\kappa<1 / 16$ we have

$$
\mathbb{P}\left\{\sup _{t \in\left[t_{0}, t_{0}+T_{0} \nu^{-1}\right]}\left|u_{\nu}^{\omega}\left(t ; u_{0}\right)\right|_{C^{m}}>K \nu^{-m \kappa}\right\} \rightarrow 1 \quad \text { as } \quad \nu \rightarrow 0,
$$

for each $t_{0} \geq 0$, if $u_{0}$ satisfies $\left|u_{0}\right|_{\infty} \leq \mathcal{K},\left|u_{0}\right|_{C^{m}} \leq \nu^{-\kappa m}$. The rate of convergence depends only on the triplet and $\kappa$.

For a proof of this result see the extended version of our work [6]. Due to (1.8), for any $m>2+n / 2$ we have

$$
\mathbb{P}\left\{\sup _{T_{0} \leq t \leq t_{0}+T_{0} \nu^{-1}}\|u(t)\|_{m} \geq K \nu^{-\left\lfloor m-\frac{n}{2}\right\rfloor \kappa}\right\} \rightarrow 1 \quad \text { as } \quad \nu \rightarrow 0
$$

for every $K>0$ and $0<\kappa<1 / 16$, where for $a \in \mathbb{R}$ we denote $\lfloor a\rfloor=\max \{n \in \mathbb{Z}: n<a\}$. This improves the first assertion of Theorem 1 for large $m$.

We have the following two corollaries from Theorem 1, valid if the Markov process defined by the equation (1.1) is mixing:

Corollary 3. Assume that $B_{m}<\infty$ for all $m$ and $b_{d} \neq 0$ for all $d$. Then eq. (1.1) is mixing and for any $\kappa<1 / 35$ and $0<\nu \leq \nu_{0}$ its unique stationary measure $\mu_{\nu}$ satisfies

$$
C^{-1} \nu^{-2 m \kappa+1} \leq \int\|u\|_{m}^{2} \mu_{\nu}(d u) \leq C \nu^{-m}, \quad 3 \leq m \in \mathbb{N} .
$$

Here $C$ and $\nu_{0}$ are as in Theorem 1.

Corollary 4. Under the assumptions of Corollary 3, for any $u_{0} \in C^{\infty}$ we have

$$
\frac{1}{2} C^{-1} \nu^{-2 m \kappa+1} \leq \mathbb{E}\left\|u\left(s ; u_{0}\right)\right\|_{m}^{2} \leq 2 C \nu^{-m}, \quad 3 \leq m \in \mathbb{N},
$$

if $s \geq T\left(\nu, u_{0}, \kappa, B_{m}, B_{m_{*}}\right)$, where $C$ is the same as in (1.9).

Theorem 1 rigorously establishes the energy cascade to high frequencies for solutions of eq. (1.1) with small $\nu$. Indeed, if $u_{0}(x)$ and $\eta(t, x)$ are smooth functions of $x$ (or even trigonometric polynomials of $x$ ), then in view of (1.7) for $0<\nu \ll 1$ and $t \gtrsim \nu^{-1}$ a substantial part of the energy $\frac{1}{2} \sum\left|u_{d}(t)\right|^{2}$ of a solution $u\left(t, x ; u_{0}\right)$ is carried by high modes $u_{d}$ with $|d| \gg 1$. Relation (1.7) (valid for all integer $m \geq 3$ ) also means that the averaged in time space-scale $l_{x}$ of solutions for (1.1) satisfies $l_{x} \in\left[\nu^{1 / 2}, \nu^{1 / 35}\right]$, and goes to zero with $\nu$ (see $[1,9]$ ). We recall that the energy cascade to high frequencies and formation of short space-scale is the driving force of the Kolmogorov theory of turbulence, see [5].

We mention that in the work [12] the stochastic CGL equation

$$
u_{t}-(\nu+i) \Delta u+i|u|^{2} u=\sqrt{\nu} \eta(t, x), \quad 0<\nu \leq 1,
$$

with linear dispersion and white in time random force $\eta$ as in (1.3) was considered under the odd periodic boundary conditions, and the inviscid limiting dynamics as $\nu \rightarrow 0$ was examined. However, since the limiting equation $(1.10)_{\nu=0}$ is a regular PDE in difference with the equation $(1.1)_{\nu=0}$, the results on the inviscid limit in [12] differ in spirit from those in our work, and we do not discuss them now.

Deterministic versions of the result of Theorem 1 for eq. (1.1) with $\eta=0$, where $\nu$ is a small non-zero complex number such that $\mathfrak{R} \nu \geq 0$ and $\mathfrak{I} \nu \leq 0$ are known, 
see [9]. In particular, if $\nu$ is a positive real number and $u_{0}$ is a smooth function of order one, then for any integer $m \geq 4$ a solution $u_{\nu}\left(t, x ; u_{0}\right)$ satisfies estimates $(1.7)$ with the averaging $\nu \mathbb{E} \int_{t}^{t+\nu^{-1}} \ldots d s$ replaced by $\nu^{1 / 3} \int_{0}^{\nu^{-1 / 3}} \ldots d s$, with the same upper bound and with the lower bound $C_{m} \nu^{-\kappa_{m} m}$, where $\kappa_{m} \rightarrow 1 / 3$ as $m \rightarrow \infty$. Moreover, it was then shown in [2] that the lower bounds remain true with $\kappa=1 / 3$, and that the estimates $\sup _{t \in\left[0,|\nu|^{-1 / 3}\right]}\|u(t)\|_{C^{m}} \geq C_{m}|\nu|^{-m / 3}, m \geq 2$, hold for smooth solutions of equation (1.1) with $\eta=0$ and any non-zero complex "viscosity" $\nu$.

The better quality of the lower bounds for solutions of the deterministic equations is due to an extra difficulty which occurs in the stochastic case: when time grows, simultaneously with increasing of high Sobolev norms of a solution, its $L_{2}$-norm may decrease, which accordingly would weaken the mechanism, responding for the energy transfer to high modes. Significant part of the proof of Theorem 1 is devoted to demonstration that the $L_{2}$-norm of a solution cannot go down without sending up the second Sobolev norm.

If $\eta=0$ and $\nu=i \delta \in i \mathbb{R}$, then (1.1) is a Hamiltonian PDE (the defocusing Schrödinger equation), and the $L_{2}$-norm is its integral of motion. If this integral is of order one, then the results of [9] (see there Appendix 3) imply that at some point of each time-interval of order $\delta^{-1 / 3}$ the $C^{m}$-norm of a corresponding solution will become $\gtrsim \delta^{-m \kappa}$ if $m \geq 2$, for any $\kappa<1 / 3$. Furthermore, if $n=2$ and $\delta=1$, then due to [4] for $m>1$ and any $M>1$ there exists a $T=T(m, M)$ and a smooth $u_{0}(x)$ such that $\left\|u_{0}\right\|_{m}<M^{-1}$ and $\left\|u\left(T ; u_{0}\right)\right\|_{m}>M$.

The paper is organized as follows. In Section 2, we recall the results from $[8,10]$ on solutions of the equation (1.1). Next we show in Section 3 that if the noise $\eta$ is non-degenerate, the $L^{2}$-norm of a solution of eq. (1.1) cannot stay too small on time intervals of order $\mathcal{O}\left(\frac{1}{\nu}\right)$ with high probability, unless its $H^{2}$-norm gets very large (see Lemma 12). Then in Section 4 we derive from this fact the assertion (1) of Theorem 1. We prove assertion (2) and both corollaries in Section 5.

Constants in estimates never depend on $\nu$, unless otherwise stated. For a metric space $M$ we denote by $\mathcal{B}(M)$ the Borel $\sigma$-algebra on $M$, and by $\mathcal{P}(M)$ - the space of probability Borel measures on $M$. By $\mathcal{D}(\xi)$ we denote the law of a r.v. $\xi$, and by $|\cdot|_{p}$ - the norm in $L_{p}\left(K^{n}\right)$.

\section{Solutions And Estimates}

Strong solutions for the equation (1.1) are defined in the usual way:

Definition 5. Let $\left(\Omega, \mathcal{F},\left\{\mathcal{F}_{t}\right\}_{t \geqslant 0}, \mathbb{P}\right)$ be the filtered probability space as in the introduction. Let $u_{0}$ in $(1.5)$ be a r.v., measurable in $\mathcal{F}_{0}$ and independent from the Wiener process $\xi$ (e.g., $u_{0}(x)$ may be a non-random function). Then a random process $u(t)=u\left(t ; u_{0}\right) \in C_{0}\left(K^{n}\right), t \in[0, T]$, adapted to the filtration, is called a strong solution of $(1.1),(1.5)$, if

(1) a.s. its trajectories $u(t)$ belong to the space

$$
\mathcal{H}([0, T]):=C\left([0, T], C_{0}\left(K^{n}\right)\right) \cap L^{2}\left([0, T], H^{1}\right) ;
$$

(2) we have

$$
u(t)=u_{0}+\int_{0}^{t}\left(\nu \Delta u-i|u|^{2} u\right) d s+\sqrt{\nu} \xi(t), \quad \forall t \in[0, T], \quad \text { a.s. },
$$

where both sides are regarded as elements of $H^{-1}$.

If (1)-(2) hold for every $T<\infty$, then $u(t)$ is a strong solution for $t \in[0, \infty)$. In this case a.s. $u \in C\left([0, \infty), C_{0}\left(K^{n}\right)\right) \cap L_{l o c}^{2}\left([0, \infty), H^{1}\right)$.

Everywhere below when we talk about solutions for the problem (1.1), (1.5) we assume that the r.v. $u_{0}$ is as in the definition above. 
The global well-posedness of eq. (1.1) was established in [8, 10]:

Theorem 6. For any $u_{0} \in C_{0}\left(K^{n}\right)$ the problem (1.1), (1.5) has a unique strong solution $u^{\omega}\left(t, x ; u_{0}\right), t \geq 0$. The family of solutions $\left\{u^{\omega}\left(t ; u_{0}\right)\right\}$ defines in the space $C_{0}\left(K^{n}\right)$ a Fellerian Markov process.

In $[8,10]$ the theorem above is proved when (1.4) is replaced by the weaker assumption $B_{*}<\infty$, where $B_{\star}=\sum\left|b_{d}\right|$ (note that $B_{*} \leq C_{n} B_{m_{*}}^{1 / 2}$ ).

The transition probability for the obtained Markov process in $C_{0}\left(K^{n}\right)$ is

$$
P_{t}(u, \Gamma)=\mathbb{P}\{u(t ; u) \in \Gamma\}, \quad u \in C_{0}\left(K^{n}\right), \Gamma \in \mathscr{B}\left(C_{0}\left(K^{n}\right)\right),
$$

and the corresponding Markov semigroup in the space $\mathscr{P}\left(C_{0}\left(K^{n}\right)\right)$ of Borel measures on $C_{0}\left(K^{n}\right)$ is formed by the operators $\left\{\mathcal{B}_{t}^{*}, t \geq 0\right\}$,

$$
\mathcal{B}_{t}^{*} \mu(\Gamma)=\int_{C_{0}\left(K^{n}\right)} P_{t}(u, \Gamma) \mu(d u), \quad t \in \mathbb{R} .
$$

Then $\mathcal{B}_{t}^{*} \mu=\mathcal{D} u\left(t ; u_{0}\right)$ if $u_{0}$ is a r.v., independent from $\xi$ and such that $\mathcal{D}\left(u_{0}\right)=\mu$.

Introducing the slow time $\tau=\nu t$ and denoting $v(\tau, x)=u\left(\frac{\tau}{\nu}, x\right)$, we rewrite eq. (1.1) in the following form, more convenient for some calculations:

$$
\frac{\partial v}{\partial \tau}-\Delta v+i \nu^{-1}|v|^{2} v=\tilde{\eta}(\tau, x)
$$

where

$$
\tilde{\eta}(\tau, x)=\frac{\partial}{\partial \tau} \tilde{\xi}(\tau, x), \quad \tilde{\xi}(\tau, x)=\sum_{d \in \mathbb{N}^{n}} b_{d} \tilde{\beta}_{d}(\tau) \varphi_{d}(x),
$$

and $\tilde{\beta}_{d}(\tau):=\nu^{1 / 2} \beta_{d}\left(\tau \nu^{-1}\right), d \in \mathbb{N}^{d}$, is another set of independent standard complex Brownian motions.

Let $\Upsilon \in C^{\infty}(\mathbb{R})$ be any smooth function such

$$
\Upsilon(r)= \begin{cases}0, & \text { for } r \leqslant \frac{1}{4} \\ r, & \text { for } r \geqslant \frac{1}{2}\end{cases}
$$

Writing $v \in \mathbb{C}$ in the polar form $v=r e^{i \Phi}$, where $r=|v|$, and recalling that $\langle\cdot, \cdot\rangle$ stands for the real scalar product in $\mathbb{C}$, we apply Itô's formula to $\Upsilon(|v|)$ and obtain that the process $\Upsilon(\tau):=\Upsilon(|v(\tau)|)$ satisfies

$$
\begin{aligned}
\Upsilon(\tau)= & \Upsilon_{0}+\int_{0}^{\tau}\left[\Upsilon^{\prime}(r)\left(\nabla r-r|\nabla \Phi|^{2}\right)\right. \\
& \left.+\frac{1}{2} \sum_{d \in \mathbb{N}^{n}} b_{d}^{2}\left(\Upsilon^{\prime \prime}(r)\left\langle e^{i \Phi}, \varphi_{d}\right\rangle^{2}+\Upsilon^{\prime}(r) \frac{1}{r}\left(\left|\varphi_{d}\right|^{2}-\left\langle e^{i \Phi}, \varphi_{d}\right\rangle^{2}\right)\right)\right] d s+\mathbb{W}(\tau),
\end{aligned}
$$

where $\Upsilon_{0}=\Upsilon(|v(0)|)$ and $\mathbb{W}(\tau)$ is the stochastic integral

$$
\mathbb{W}(\tau)=\sum_{d \in \mathbb{N}^{n}} \int_{0}^{\tau} \Upsilon^{\prime}(r) b_{d} \varphi_{d}\left\langle e^{i \Phi}, d \tilde{\beta}_{d}(s)\right\rangle .
$$

In [10] eq. (2.1) is considered with $\nu=1$ and, following [8], the norm $|v(t)|_{\infty}$ of a solution $v$ is estimated via $\Upsilon(t)$ (since $|v| \leq \Upsilon+1 / 2$ ). But the nonlinear term $i \nu^{-1}|v|^{2} v$ does not contribute to eq. (2.2), which is the same as the $\Upsilon$-equation (2.3) in [10] (and as the corresponding equation in [8, Section 3.1]). So the estimates on $|\Upsilon(t)|_{\infty}$ and the resulting estimates on $|v(t)|_{\infty}$, obtained in [10], remain true for solutions of (2.1) with any $\nu$. Thus we get the following upper bound for quadratic exponential moments of the $L_{\infty}$-norms of solutions: ${ }^{2}$

\footnotetext{
${ }^{2}$ In [8] polynomial moments of the random variables $\left.\sup _{\tau \leqslant s \leqslant \tau+T}|v(s)|_{\infty}^{2}\right)$ are estimated, and in [10] these results are strengthened to the exponential bounds $(2.3)$.
} 
Theorem 7. For any $T>0$ there are constants $c_{*}>0$ and $C>0$, depending only on $B_{*}$ and $T$, such that for any r.v. $v_{0}^{\omega} \in C_{0}\left(K^{n}\right)$ as in Definition 5 , any $\tau \geqslant 0$ and any $c \in\left(0, c_{*}\right]$, a solution $v\left(\tau ; v_{0}\right)$ of eq. (2.1) satisfies

$$
\mathbb{E} \exp \left(c \sup _{\tau \leqslant s \leqslant \tau+T}|v(s)|_{\infty}^{2}\right) \leqslant C \mathbb{E} \exp \left(5 c\left|v_{0}\right|_{\infty}^{2}\right) \leq \infty .
$$

In $[10]$ the result above is proved for a deterministic initial data $v_{0}$. The theorem's assertion follows by averaging the result of [10] in $v_{0}^{\omega}$.

The estimate (2.3) is crucial for derivation of further properties of solutions, including the given below upper bounds for their Sobolev norms, obtained in the work [8]. Since the scaling of the equation in [8] differs from that in (2.1) and the result there is a bit less general than in the theorem below, a sketch of the proof is given in Appendix B.

Theorem 8. Assume that $B_{m}<\infty$ for some $m \in \mathbb{N}$, and $v_{0}=v_{0}^{\nu} \in H^{m} \cap C_{0}\left(K^{n}\right)$ satisfies

$$
\left|v_{0}\right|_{\infty} \leq M, \quad\left\|v_{0}\right\|_{m} \leq M_{m} \nu^{-m}, \quad 0<\nu \leq 1
$$

Then

$$
\mathbb{E}\left\|v\left(\tau ; v_{0}\right)\right\|_{m}^{2} \leqslant C_{m} \nu^{-m}, \quad \forall \tau \in[0, \infty),
$$

where $C_{M, m}$ also depends on $M, M_{m}$ and $B_{m}, B_{m_{*}}$.

Neglecting the dependence on $\nu$, we have that if $B_{m}<\infty, m \in \mathbb{N}$, and a r.v. $v_{0}^{\omega} \in H^{m} \cap C_{0}\left(K^{n}\right)$ satisfies $\mathbb{E}\left\|v_{0}\right\|_{m}^{2}<\infty$ and $\mathbb{E} \exp \left(c\left|v_{0}\right|_{\infty}^{2}\right)<\infty$ for some $c>0$, then eq. (2.1) has a solution, equal $v_{0}$ at $t=0$, such that

$$
\begin{gathered}
\mathbb{E}\left\|v\left(\tau ; v_{0}\right)\right\|_{m}^{2} \leq e^{-t} \mathbb{E}\left\|v_{0}\right\|_{m}^{2}+C, \quad \tau \geq 0, \\
\mathbb{E} \sup _{0 \leq \tau \leq T}\left\|v\left(\tau ; v_{0}\right)\right\|_{m}^{2} \leq C^{\prime},
\end{gathered}
$$

where $C>0$ depend on $c, \nu, \mathbb{E} \exp \left(c\left|v_{0}\right|_{\infty}^{2}\right), B_{m_{*}}$ and $B_{m}$, while $C^{\prime}$ also depends on $\mathbb{E}\left\|v_{0}\right\|_{m}^{2}<\infty$ and $T$. See Appendix B.

As it is shown in [10], the estimate (2.3) jointly with an abstract theorem from [11], imply that under a mild nondegeneracy assumption on the random force the Markov process in the space $C_{0}\left(K^{n}\right)$, constructed in Theorem 6 , is mixing:

Theorem 9. For each $\nu>0$, there is an integer $N=N\left(B_{*}, \nu\right)>0$ such that if $b_{d} \neq 0$ for $|d| \leqslant N$, then the equation (1.1) is mixing. I.e. it has a unique stationary measure $\mu_{\nu} \in \mathscr{P}\left(C_{0}\left(K^{n}\right)\right)$, and for any probability measure $\lambda \in \mathscr{P}\left(C_{0}\left(K^{n}\right)\right)$ we have $\mathcal{B}_{t}^{*} \lambda \rightarrow \mu_{\nu}$ as $t \rightarrow \infty$.

Under the assumption of Theorem 8, for any $u_{0} \in H^{m}$ the law $\mathcal{D} u\left(t ; u_{0}\right)$ of a solution $u\left(t ; u_{0}\right)$ is a measure in $H^{m}$. The mixing property in Theorem 9 and (2.4) easily imply

Corollary 10. If under the assumptions of Theorem $9 B_{m}<\infty$ for some $m \in \mathbb{N}$ and $u_{0} \in H^{m}$, then $\mathcal{D}\left(u\left(t ; u_{0}\right)\right) \rightarrow \mu_{\nu}$ in $\mathcal{P}\left(H^{m}\right)$.

In view of Theorems 7,8 with $v_{0}=0$ and the established mixing, we have:

Corollary 11. Under the assumptions of Theorem 9, if $v^{\text {st }}(\tau)$ is the stationary solution of the equation, then

$$
\mathbb{E} \exp \left(c_{*} \sup _{\tau \leqslant s \leqslant \tau+T}\left|v^{s t}(s)\right|_{\infty}^{2}\right) \leqslant \mathcal{C}
$$

where the constant $\mathcal{C}>0$ depends only on $T$ and $B_{*}$. If in addition $B_{m}<\infty$ for some $m \in \mathbb{N} \cup\{0\}$, then $\mathbb{E}\left\|v^{\text {st }}(\tau)\right\|_{m}^{2} \leqslant C_{m} \nu^{-m}$, where $C_{m}$ depends on $B_{*}$ and $B_{m}$. 
Finally we note that applying Itô's formula to $\left\|v^{s t}(\tau)\right\|_{0}^{2}$, where $v^{s t}$ is a stationary solution of (2.1), and taking the expectation we get the balance relation

$$
\mathbb{E}\left\|v^{s t}(\tau)\right\|_{1}^{2}=B_{0}
$$

We cannot prove that $\mathbb{E}\left\|v^{s t}(\tau)\right\|_{0}^{2} \geq B^{\prime}>0$ for some $\nu$-independent constant $B^{\prime}$, and cannot bound from below the energy $\frac{1}{2} \mathbb{E}\left\|v\left(\tau ; v_{0}\right)\right\|_{0}^{2}$ of a solution $v$ by a positive $\nu$-independent quantity. Instead in next section we get a weaker conditional lower bound on the energies of solutions.

\section{Conditional LOWER BOUnd FOR The $L^{2}$-NORM OF SOlutions}

In this section we prove the following result:

Lemma 12. Let $B_{2}<\infty$ and $u\left(\tau ; u_{0}\right)$, where $u_{0} \in H^{2} \cap C_{0}\left(K^{n}\right)$ is non-random, be a solution of eq. (2.1). Take any constants $\chi>0, \Gamma \geq 1, \tau_{0} \geq 0$, and define the stopping time

$$
\tau_{\Gamma}:=\inf \left\{\tau \geq \tau_{0}:\|u(\tau)\|_{2} \geqslant \Gamma\right\}
$$

(as usual, $\tau_{\Gamma}=\infty$ if the set under the inf-sign is empty). Then

$$
\mathbb{E} \int_{\tau_{0}}^{\tau \wedge \tau_{\Gamma}} \mathbb{I}_{[0, \chi]}\left(\|u(s)\|_{0}\right) d s \leqslant 2\left(1+\tau-\tau_{0}\right) B_{0}^{-1} \chi \Gamma,
$$

for any $\tau>\tau_{0}$.

Proof. We establish the result by adapting the proof from [16] (also see [11, Theorem 5.2.12]) to non-stationary solutions. The argument relies on the concept of local time for semi-martingales (see e.g. [14, Chapter VI.1] for details of the concept). By $[\cdot]_{b}$ we denote the quasinorm $[u]_{b}^{2}=\sum_{d}\left|u_{d}\right|^{2} b_{d}^{2}$.

Without loss of generality we assume $\tau_{0}=0$. Otherwise we just need to replace $u(\tau, x)$ by the process $\tilde{u}(\tau, x):=u\left(\tau+\tau_{0}, x\right)$, apply the lemma with $\tau_{0}=0$ and with $u_{0}$ replaced by the initial data $\tilde{u}_{0}^{\omega}=u^{\omega}\left(\tau_{0} ; u_{0}\right)$, and then average the estimate in the random $\tilde{u}_{0}^{\omega}$.

Let us write the solution $u\left(\tau ; u_{0}\right)$ as $u(\tau)=\sum_{d \in \mathbb{N}^{n}} u_{d}(\tau) \varphi_{d}$. For any fixed function $g \in C^{2}(\mathbb{R})$, consider the process

$$
f(\tau)=g\left(\left\|u\left(\tau \wedge \tau_{\Gamma}\right)\right\|_{0}^{2}\right)
$$

Since

$$
\left.\partial_{u} g\left(\|u\|_{0}^{2}\right)=2 g^{\prime}\left(\|u\|_{0}^{2}\right)\langle\langle u, \cdot\rangle\rangle, \quad \partial_{u u} g\left(\|u\|_{0}^{2}\right)=4 g^{\prime \prime}\left(\|u\|_{0}^{2}\right)\langle\langle u, \cdot\rangle\rangle\langle u, \cdot\rangle\right\rangle+2 g^{\prime}\left(\|u\|_{0}^{2}\right)\langle\langle\cdot, \cdot\rangle\rangle,
$$

then by Itô's formula we have

$$
f(\tau)=f(0)+\int_{0}^{\tau \wedge \tau_{\Gamma}} A(s) d s+\sum_{d \in \mathbb{N}^{n}} b_{d} \int_{0}^{\tau \wedge \tau_{\Gamma}} 2 g^{\prime}\left(\|u(s)\|_{0}^{2}\right)\left\langle u_{d}(s), d \beta_{d}(s)\right\rangle,
$$

where

$$
\begin{aligned}
A(s) & =2 g^{\prime}\left(\|u\|_{0}^{2}\right)\left\langle\left\langle u, \Delta u-\frac{1}{\nu} i|u|^{2} u\right\rangle\right\rangle+2 \sum_{d} b_{d}^{2}\left(g^{\prime \prime}\left(\|u\|_{0}^{2}\right)\left|u_{d}\right|^{2}+g^{\prime}\left(\|u\|_{0}^{2}\right)\right) \\
& =-2 g^{\prime}\left(\|u\|_{0}^{2}\right)\|u\|_{1}^{2}+2 g^{\prime \prime}\left(\|u\|_{0}^{2}\right)[u]_{b}^{2}+2 g^{\prime}\left(\|u\|_{0}^{2}\right) B_{0}, \quad u=u(s) .
\end{aligned}
$$

Step 1: We firstly show that for any bounded measurable set $G \subset \mathbb{R}$, denoting by $\mathbb{I}_{G}$ its indicator function, we have the following equality

$$
\begin{aligned}
& 2 \mathbb{E} \int_{0}^{\tau \wedge \tau_{\Gamma}} \mathbb{I}_{G}(f(s))\left(g^{\prime}\left(\|u(s)\|_{0}^{2}\right)\right)^{2}[u(s)]_{b}^{2} d s=\int_{-\infty}^{\infty} \mathbb{I}_{G}(a) \\
& {\left[\mathbb{E}(f(\tau)-a)_{+}-\mathbb{E}(f(0)-a)_{+}-\mathbb{E} \int_{0}^{\tau \wedge \tau_{\Gamma}} \mathbb{I}_{(a+\infty)}(f(s)) A(s) d s\right] d a . }
\end{aligned}
$$


Let $L(\tau, a),(\tau, a) \in[0, \infty) \times \mathbb{R}$, be the local time for the semi martingale $f(\tau)$ (see e.g. [14, Chapter VI.1]). Since in view of (3.2) the quadratic variation of the process $f(\tau)$ is

$$
d\langle f, f\rangle_{s}=\sum_{d}\left(2 g^{\prime}\left(\|u\|_{0}^{2}\right)\left|u_{d}\right| b_{d}\right)^{2}=4\left(g^{\prime}\left(\|u\|_{0}^{2}\right)\right)^{2}[u]_{b}^{2},
$$

then for any bounded measurable set $G \subset \mathbb{R}$, we have the following equality (known as the occupation time formula, see [14, Corollary VI.1.6]),

$$
\int_{0}^{\tau \wedge \tau_{\Gamma}} \mathbb{I}_{G}(f(s)) 4\left(g^{\prime}\left(\|u(s)\|_{0}^{2}\right)\right)^{2}[u(s)]_{b}^{2} d s=\int_{-\infty}^{\infty} \mathbb{I}_{G}(a) L(\tau, a) d a .
$$

For the local time $L(\tau, a)$, due to Tanaka's formula (see [14, Theorem VI.1.2]) we have

$$
\begin{aligned}
(f(\tau)-a)_{+}= & (f(0)-a)_{+} \\
& +\sum_{d \in \mathbb{N}^{n}} b_{d} \int_{0}^{\tau \wedge \tau_{\Gamma}} \mathbb{I}_{(a,+\infty)}(f(s)) 2 g^{\prime}\left(\|u(s)\|_{0}^{2}\right)\left\langle u_{d}(s), d \beta_{d}(s)\right\rangle \\
& +\int_{0}^{\tau \wedge \tau_{\Gamma}} \mathbb{I}_{(a,+\infty)}(f(s)) A(s) d s+\frac{1}{2} L(\tau, a) .
\end{aligned}
$$

Taking expectation of both sides of (3.5) and (3.6) we obtain the required equality (3.4).

Step 2: Let us choose $G=\left[\rho_{0}, \rho_{1}\right]$ with $\rho_{1}>\rho_{0}>0$, and $g(x)=g_{\rho_{0}}(x) \in C^{2}(\mathbb{R})$ such that $g^{\prime}(x) \geqslant 0, g(x)=\sqrt{x}$ for $x \geqslant \rho_{0}$ and $g(x)=0$ for $x \leqslant 0$. Then due to the factors $\mathbb{I}_{G}(f)$ and $\mathbb{I}_{G}(a)$ in $(3.4)$, we may there replace $g(x)$ by $\sqrt{x}$, and accordingly replace $g\left(\|u\|_{0}^{2}\right), g^{\prime}\left(\|u\|_{0}^{2}\right)$ and $g^{\prime \prime}\left(\|u\|_{0}^{2}\right)$ by $\|u\|_{0}, \frac{1}{2}\|u\|_{0}^{-1}$ and $-\frac{1}{4}\|u\|_{0}^{-3}$. So the relation (3.4) takes the form

$$
\begin{aligned}
& \mathbb{E} \int_{0}^{\tau \wedge \tau_{\Gamma}} \mathbb{I}_{G}(f(s))\|u(s)\|_{0}^{-2}[u(s)]_{b}^{2}=2 \int_{\rho_{0}}^{\rho_{1}}\left[\mathbb{E}(f(\tau)-a)_{+}-\mathbb{E}(f(0)-a)_{+}\right] d a \\
& -2 \int_{\rho_{0}}^{\rho_{1}}\left\{\mathbb{E} \int_{0}^{\tau \wedge \tau_{\Gamma}} \mathbb{I}_{(a,+\infty)}(f(s))\left[\frac{2}{2\|u(s)\|_{0}}\left(B_{0}-\|u(s)\|_{1}^{2}\right)-\frac{2}{4\|u(s)\|_{0}^{3}}[u(s)]_{b}^{2}\right] d s\right\} d a .
\end{aligned}
$$

Since the l.h.s. of the above equality is non-negative, we have

$$
\begin{aligned}
& \int_{\rho_{0}}^{\rho_{1}}\left[\mathbb{E} \int_{0}^{\tau \wedge \tau_{\Gamma}} \mathbb{I}_{(a,+\infty)}(f(s)) \frac{1}{\|u(s)\|_{0}^{3}}\left(B_{0}\|u(s)\|_{0}^{2}-\frac{1}{2}[u(s)]_{b}^{2}\right) d s\right] d a \\
& \leqslant \int_{\rho_{0}}^{\rho_{1}} \mathbb{E}\left[\left((f(\tau)-a)_{+}-(f(0)-a)_{+}\right)+\int_{0}^{\tau \wedge \tau_{\Gamma}} \mathbb{I}_{(a,+\infty)}(f(s)) \frac{\|u(s)\|_{1}^{2}}{\|u(s)\|_{0}} d s\right] d a .
\end{aligned}
$$

Noting that

$$
B_{0}\|u\|_{0}^{2}-\frac{1}{2}[u(s)]_{b}^{2}=\sum_{d \in \mathbb{N}^{n}}\left(B_{0}-\frac{1}{2} b_{d}^{2}\right)\left|u_{d}\right|^{2} \geqslant \frac{B_{0}}{2}\|u\|_{0}^{2},
$$

that by the definition of the stopping time $\tau_{\Gamma}$

$$
(f(\tau)-a)_{+}-(f(0)-a)_{+} \leqslant \Gamma,
$$

and that by interpolation,

$$
\int_{0}^{\tau \wedge \tau_{\Gamma}} \frac{\|u(s)\|_{1}^{2}}{\|u(s)\|_{0}} d s \leqslant \int_{0}^{\tau \wedge \tau_{\Gamma}}\|u(s)\|_{2} d s \leqslant\left(\tau \wedge \tau_{\Gamma}\right) \Gamma,
$$

we derive from (3.7) the relation

$$
\frac{B_{0}}{2} \int_{\rho_{0}}^{\rho_{1}}\left(\mathbb{E} \int_{0}^{\tau \wedge \tau_{\Gamma}} \mathbb{I}_{(a,+\infty)}(f(s))\|u(s)\|_{0}^{-1} d s\right) d a \leq\left(\rho_{1}-\rho_{0}\right) \Gamma(1+\tau) .
$$


When $\rho_{0} \rightarrow 0$, we have $g(x) \rightarrow \sqrt{x}$ and $f(\tau) \rightarrow\left\|u\left(\tau \wedge \tau_{\Gamma}\right)\right\|_{0}$. So sending $\rho_{0}$ to 0 and using Fatou's lemma we get from the last estimate that

$$
\int_{0}^{\rho_{1}} \mathbb{E} \int_{0}^{\tau \wedge \tau_{\Gamma}} \mathbb{I}_{(a, \infty)}\left(\|u(s)\|_{0}\right)\|u(s)\|_{0}^{-1} d s d a \leqslant 2 \rho_{1}(1+\tau) B_{0}^{-1} \Gamma .
$$

As the l.h.s. above is not smaller than

$$
\frac{1}{\chi} \int_{0}^{\rho_{1}} \mathbb{E} \int_{0}^{\tau \wedge \tau_{\Gamma}} \mathbb{I}_{(a, \chi]}\left(\|u(s)\|_{0}\right) d s d a,
$$

then

$$
\frac{1}{\rho_{1}} \int_{0}^{\rho_{1}} \mathbb{E} \int_{0}^{\tau \wedge \tau_{\Gamma}} \mathbb{I}_{(a, \chi]}\left(\|u(s)\|_{0}\right) d s d a \leqslant 2(1+\tau) B_{0}^{-1} \Gamma \chi .
$$

By the monotone convergence theorem

$$
\lim _{a \rightarrow 0} \mathbb{E} \int_{0}^{\tau \wedge \tau_{\Gamma}} \mathbb{I}_{(a, \chi]}\left(\|u(s)\|_{0}\right) d s=\mathbb{E} \int_{0}^{\tau \wedge \tau_{\Gamma}} \mathbb{I}_{(0, \chi]}\left(\|u(s)\|_{0}\right) d s,
$$

so we get from (3.8) that

$$
\mathbb{E} \int_{0}^{\tau \wedge \tau_{\Gamma}} \mathbb{I}_{(0, \chi]}\left(\|u(s)\|_{0}\right) d s \leqslant 2(1+\tau) B_{0}^{-1} \Gamma \chi .
$$

Step 3: We continue to verify that

$$
\mathbb{E} \int_{0}^{\tau \wedge \tau_{\Gamma}} \mathbb{I}_{\{0\}}\left(\|u(s)\|_{0}\right) d s=0 .
$$

To do this let us fix any index $d \in \mathbb{N}^{n}$ such that $b_{d} \neq 0$. The process $u_{d}(\tau)$ is a semimartingale, $d u_{d}=v_{d} d s+b_{d} d \beta_{d}$, where $v_{d}(s)$ is the $d$-th Fourier coefficient of $\Delta u+\frac{1}{\nu} i|u|^{2} u$ for the solution $u(\tau)=\sum_{d} u_{d}(\tau) \varphi_{d}$ which we discuss. Consider the stopping time

$$
\tau_{R}=\inf \left\{s \leq \tau \wedge \tau_{\Gamma}:|u(s)|_{\infty} \geq R\right\} .
$$

Due to (2.3) and (2.6), $\mathbb{P}\left(\tau_{R}=\tau \wedge \tau_{\Gamma}\right) \rightarrow 1$ as $R \rightarrow \infty$. Let us denote $u_{d}^{R}(\tau)=$ $u_{d}\left(\tau \wedge \tau_{R}\right)$. To prove (3.10) it suffices to verify that

$$
\pi(\delta):=\mathbb{E} \int_{0}^{\tau \wedge \tau_{\Gamma}} \mathbb{I}_{\left\{\left|u_{d}(s)\right|<\delta\right\}} d s \rightarrow 0 \quad \text { as } \quad \delta \rightarrow 0 .
$$

If we replace above $u_{d}$ by $u_{d}^{R}$, then the obtained new quantity $\pi^{R}(\delta)$ differs from $\pi(\delta)$ at most by $\mathbb{P}\left(\tau_{R}<\tau \wedge \tau_{\Gamma}\right)$. The process $u_{d}^{R}$ is an Ito process with a bounded drift. So by [7, Theorem 2.2.2, p. 52], $\pi^{R}(\delta)$ goes to zero with $\delta$. Thus, given any $\varepsilon>0$, we firstly choose $R$ sufficiently big and then $\delta$ sufficiently small to achieve $\pi(\delta)<\varepsilon$, for a suitable $\delta(\varepsilon)>0$. So (3.10) is verified. Jointly with (3.9) this proves (3.1).

\section{LOWER BOUNDS FOR SOBOLEV NORMS OF SOLUTIONS}

In this section we work with eq. (1.1) in the original time scale $t$ and provide lower bounds for the $H^{m}$-norms of its solutions with $m>2$. This will prove the assertion (1) of Theorem 1. As always, the constants do not depend on $\nu$, unless otherwise stated.

Theorem 13. For any integer $m \geqslant 3$, if $B_{m}<\infty$ and

$$
0<\kappa<\frac{1}{35}, \quad T_{0} \geq 0, \quad T_{1}>0,
$$

then for any r.v. $u_{0}(x) \in H^{m} \cap C_{0}\left(K^{n}\right)$, satisfying

$$
\mathbb{E}\left\|u_{0}\right\|_{m}^{2}<\infty, \quad \mathbb{E} \exp \left(c\left|u_{0}\right|_{\infty}^{2}\right) \leq C<\infty
$$

for some $c, C>0$, we have

$$
\mathbb{P}\left\{\sup _{T_{0} \leq t \leq T_{0}+T_{1} \nu^{-1}}\left\|u\left(t ; u_{0}\right)\right\|_{m} \geq K \nu^{-m \kappa}\right\} \rightarrow 1 \quad \text { as } \quad \nu \rightarrow 0,
$$


for every $K>0$.

Proof. Consider the complement to the event in (4.2):

$$
Q^{\nu}=\left\{\sup _{T_{0} \leqslant t \leqslant T_{0}+\frac{T_{1}}{\nu}}\|u(t)\|_{m}<K \nu^{-m \kappa}\right\} .
$$

We will prove the assertion (4.2) by contradiction. Namely, we assume that there exists a $\gamma>0$ and a sequence $\nu_{j} \rightarrow 0$ such that

$$
\mathbb{P}\left(Q^{\nu_{j}}\right) \geqslant 5 \gamma \text { for } j=1,2, \ldots,
$$

and will derive a contradiction. Below we write $Q^{\nu_{j}}$ as $Q$ and always suppose that

$$
\nu \in\left\{\nu_{1}, \nu_{2}, \ldots\right\} \text {. }
$$

The constants in the proof may depend on $\mathcal{K}, K, \gamma, B_{m \vee m_{*}}$, but not on $\nu$.

Without lost of generality we assume that $T_{1}=1$. For any $T_{0}>0$, due to (2.5) and (2.3) the r.v. $\tilde{u}_{0}:=u\left(T_{1}\right)$ satisfies $(4.1)$ with $c$ replaced by $c / 5$. So considering $\tilde{u}(t, x)=u\left(t+T_{0}, x\right)$ we may assume that $T_{0}=0$.

Let us denote $J_{1}=\left[0, \frac{1}{\nu}\right]$. Due to Theorem 7 ,

$$
\mathbb{P}\left(Q_{1}\right) \geqslant 1-\gamma, \quad Q_{1}=\left\{\sup _{t \in J_{1}}|u(t)|_{\infty} \leqslant C_{1}(\gamma)\right\},
$$

uniformly in $\nu$, for a suitable $C_{1}(\gamma)$. Then, by the definition of $Q$ and Sobolev's interpolation,

$$
\left\|u^{\omega}(t)\right\|_{l} \leqslant C_{l, \gamma} \nu^{-l \kappa}, \quad \omega \in Q \cap Q_{1}, t \in J_{1},
$$

for $l \in[0, m]$ (and any $\nu \in\left\{\nu_{1}, \nu_{2}, \ldots\right\}$ ).

Denote $J_{2}=\left[0, \frac{1}{2 \nu}\right]$ and consider the stopping time

$$
\tau_{1}=\inf \left\{t \in J_{2}:\|u(t)\|_{2} \geqslant C_{2, \gamma} \nu^{-2 \kappa}\right\} \leq \frac{1}{2 \nu} .
$$

Then $\tau_{1}=\frac{1}{2 \nu}$ for $\omega \in Q \cap Q_{1}$. So due to (3.1) with $\Gamma=C_{2, \gamma} \nu^{-2 \kappa}$, for any $\chi>0$, we have

$$
\begin{aligned}
\mathbb{E}\left(\nu \int_{J_{2}} \mathbb{I}_{[0, \chi]}\left(\|u(s)\|_{0}\right) d s \mathbb{I}_{Q \cap Q_{1}}(\omega)\right) & =\mathbb{E}\left(\nu \int_{0}^{\frac{1}{2 \nu} \wedge \tau_{1}} \mathbb{I}_{[0, \chi]}\left(\|u(s)\|_{0}\right) d s \mathbb{I}_{Q \cap Q_{1}}(\omega)\right) \\
& \leqslant \mathbb{E}\left(\nu \int_{0}^{\frac{1}{2 \nu} \wedge \tau_{1}} \mathbb{I}_{[0, \chi]}\left(\|u(s)\|_{0}\right) d s\right) \leqslant C \nu^{-2 \kappa} \chi .
\end{aligned}
$$

Consider the event

$$
\Lambda=\left\{\omega \in Q \cap Q_{1}:\|u(s)\|_{0} \leqslant \chi, \forall s \in J_{2}\right\} .
$$

Due to the above, we have,

$$
\mathbb{P}(\Lambda) \leqslant 2 \mathbb{E}\left(\nu \int_{J_{2}} \mathbb{I}_{[0, \chi]}\left(\|u(s)\|_{0}\right) d s \mathbb{I}_{Q \cap Q_{1}}(\omega)\right) \leqslant 2 C \nu^{-2 \kappa} \chi
$$

So $\mathbb{P}(\Lambda) \leqslant \gamma$ if we choose

$$
\chi=c_{3}(\gamma) \nu^{2 \kappa}, \quad c_{3}(\gamma)=\gamma(2 C)^{-1} .
$$

Let us set

$$
Q_{2}=\left(Q \cap Q_{1}\right) \backslash \Lambda, \quad \mathbb{P}\left(Q_{2}\right) \geqslant 3 \gamma,
$$

and for $\chi$ as in (4.5), consider the stopping time

$$
\tilde{\tau}_{1}=\inf \left\{t \in J_{2}:\|u(t)\|_{0} \geqslant \chi\right\} .
$$

Then $\tilde{\tau}_{1} \leqslant \frac{1}{2 \nu}$ for all $\omega \in Q_{2}$. Consider the function

$$
v(t, x):=u\left(\tilde{\tau}_{1}+t, x\right), \quad t \in\left[0, \frac{1}{2 \nu}\right] .
$$

It solves eq. (1.1) with modified Wiener processes and with initial data $v_{0}(x)=$ $u^{\omega}\left(\tilde{\tau}_{1}, x\right)$, satisfying

$$
\left\|v_{0}^{\omega}\right\|_{0} \geq \chi=c \nu^{2 \kappa} \quad \text { if } \quad \omega \in Q_{2} .
$$


Now we introduce another stopping time, in terms of $v(t, x)$ :

$$
\tau_{2}=\inf \left\{t \in\left[0, \frac{1}{2 \nu}\right]:\|v(t)\|_{m} \geqslant K \nu^{-m \kappa}\right\} \leq \frac{1}{2 \nu} .
$$

For $\omega \in Q_{2}, \tau_{2}=\frac{1}{2 \nu}$ and in view of (4.4)

$$
\left\|v^{\omega}(t)\right\|_{l} \leqslant C_{3}(\gamma) \nu^{-l \kappa}, \quad t \in\left[0, \frac{1}{2 \nu}\right], l \in[0, m], \quad \forall \omega \in Q_{2} .
$$

Step 1: Let us estimate from above the increment $\mathscr{E}(t, x)=\left|v\left(t \wedge \tau_{2}, x\right)\right|^{2}-\left|v_{0}(x)\right|^{2}$. Due to Itô's formula, we have that

$$
\begin{gathered}
\mathscr{E}(t, x)=2 \nu \int_{0}^{t \wedge \tau_{2}}\left(\langle v(s, x), \Delta v(s, x)\rangle+\sum_{d \in \mathbb{N}^{n}} b_{d}^{2} \varphi_{d}^{2}(x)\right) d s+\sqrt{\nu} M(t, x), \\
M(t, x)=\int_{0}^{t \wedge \tau_{2}} \sum_{d \in \mathbb{N}^{n}} b_{d} \varphi_{d}(x)\left\langle v(s, x), d \beta_{d}(s)\right\rangle .
\end{gathered}
$$

We treat $M$ as a martingale $M(t)$ in the space $H^{1}$. Since in view of (A.3) for $0 \leq s<\tau_{2}$ we have

$$
\left\|v(s) \varphi_{d}\right\|_{1} \leq C\left(|v(s)|_{\infty}\left\|\varphi_{d}\right\|_{1}+\left.\left|v(s) \|_{1}\right| \varphi_{d}\right|_{\infty}\right) \leq C\left(\zeta d+\zeta^{(m-1) / m} \nu^{-\kappa}\right),
$$

where $\zeta=\sup _{0 \leqslant s \leqslant \frac{1}{\nu}}|u(s)|_{\infty}$ (the assertion is empty if $\tau_{2}=0$ ), then for any $0<T_{\star} \leq \frac{1}{2 \nu}$

$$
\mathbb{E}\left\|M\left(T_{*}\right)\right\|_{1}^{2} \leq \int_{0}^{T_{*}} \mathbb{E} \sum_{d} b_{d}^{2}\left\|\varphi_{d} v(s)\right\|_{1}^{2} d s \leq C T_{*} \nu^{-2 \kappa},
$$

where we used that $B_{1}<\infty$. So by Doob's inequality

$$
\mathbb{P}\left(\sup _{0 \leqslant s \leqslant T_{*}}\|M(s)\|_{1}^{2} \geqslant r^{2}\right) \leqslant C T_{*} r^{-2} \nu^{-2 \kappa}, \quad \forall r>0 .
$$

Let us choose

$$
T_{*}=\nu^{-b}, \quad b \in(0,1),
$$

where $b$ will be specified later. Then $1 \leq T_{*} \leq \frac{1}{2 \nu}$ if $\nu$ is sufficiently small, so due to $(4.10)$

$$
\mathbb{P}\left(Q_{3}\right) \geqslant 1-\gamma, \quad Q_{3}=\left\{\sup _{0 \leqslant \tau \leqslant T_{*}}\|M(\tau)\|_{1} \leqslant C_{4}(\gamma) \nu^{-\kappa} \sqrt{T_{*}}\right\},
$$

for a suitable $C_{4}(\gamma)$ (and for $\nu \ll 1$ ); thus $\mathbb{P}\left(Q_{2} \cap Q_{3}\right) \geq 2 \gamma$. Since $\|\langle v, \Delta v\rangle\|_{1} \leq$ $C|v|_{\infty}\|v\|_{3}$ by (A.2) and $\left\|\sum_{d} b_{d} \varphi_{d}\right\|_{1} \leq C$, then in view of (4.8) and the definition of $Q_{3}$,

$$
\left\|\mathscr{E}^{\omega}(\tau)\right\|_{1} \leqslant C(\gamma)\left(\nu^{1-3 \kappa} T_{*}+\nu^{\frac{1}{2}-\kappa} T_{*}^{1 / 2}\right), \quad \forall \tau \in\left[0, T_{*}\right], \quad \forall \omega \in Q_{2} \cap Q_{3} .
$$

Step 2: For any $x \in K^{n}$, denoting $R(t)=|v(t, x)|^{2}, a(t)=\Delta v(t, x)$ and $\xi(t)=$ $\xi(t, x)$, we write the equation for $v(t):=v(t, x)$ as an Itô process:

$$
d v(t)=(-i R v+\nu a) d t+\sqrt{\nu} d \xi(t) .
$$

Setting $w(t)=e^{i \int_{0}^{t} R(s) d s} v(t)$, we observe that $w$ also is an Itô process, $w(0)=v_{0}$ and $d v=e^{-i \int_{0}^{t} R(s) d s} d w-i R v d t$. From here and (4.12),

$$
w(t)=v_{0}+\nu \int_{0}^{t} e^{i \int_{0}^{s} R\left(s^{\prime}\right) d s^{\prime}} a(s) d s+\sqrt{\nu} \int_{0}^{t} e^{i \int_{0}^{s} R\left(s^{\prime}\right) d s^{\prime}} d \xi(s) .
$$

So $v\left(t \wedge \tau_{2}\right)=v\left(t \wedge \tau_{2}, x\right)$ can be written as

$$
v\left(t \wedge \tau_{2}, x\right)=I_{1}\left(t \wedge \tau_{2}, x\right)+I_{2}\left(t \wedge \tau_{2}, x\right)+I_{3}\left(t \wedge \tau_{2}, x\right),
$$

where

$$
\begin{array}{r}
I_{1}(t, x)=e^{-i \int_{0}^{t}|v(s, x)|^{2} d s} v_{0}, \quad I_{2}(t, x)=\nu \int_{0}^{t} e^{-i \int_{s}^{t}\left|v\left(s^{\prime}, x\right)\right|^{2} d s^{\prime}} \Delta v(s, x) d s, \\
I_{3}(t, x)=\sqrt{\nu} e^{-i \int_{0}^{t}\left|v\left(s^{\prime}, x\right)\right|^{2} d s^{\prime}} \int_{0}^{t} e^{i \int_{0}^{s}\left|v\left(s^{\prime}, x\right)\right|^{2} d s^{\prime}} d \xi(s, x) .
\end{array}
$$


Our next goal is to obtain a lower bound for $\left\|v\left(T_{*}\right)\right\|_{1}$ when $\omega \in Q_{2} \cap Q_{3}$, using the above decomposition (4.13).

Step 3: We first deal with the stochastic term $I_{3}(t)$. For $0 \leqslant s \leqslant s_{1} \leqslant T_{\star} \wedge \tau_{2}$ we set

$$
W\left(s, s_{1}, x\right):=\exp \left(i \int_{s}^{s_{1}}\left|v\left(s^{\prime}, x\right)\right|^{2} d s^{\prime}\right), \quad F\left(s, s_{1}, x\right):=\int_{s}^{s_{1}}\left|v\left(s^{\prime}, x\right)\right|^{2} d s^{\prime} ;
$$

then $W\left(s, s_{1}, x\right)=\exp \left(i F\left(s, s_{1}, x\right)\right)$. The functions $F$ and $W$ are periodic in $x$, but not odd. Speaking about them we understand $\|\cdot\|_{m}$ as the non-homogeneous Sobolev norm, so $\|F\|_{m}^{2}=\|F\|_{0}^{2}+\left\|(-\Delta)^{m / 2} F\right\|_{0}^{2}$, etc. We write $I_{3}$ as

$$
I_{3}(t)=\sqrt{\nu} \bar{W}\left(0, t \wedge \tau_{2}, x\right) \int_{0}^{t \wedge \tau_{2}} W(0, s, x) d \xi(s, x) .
$$

In view of (A.1),

$$
\left\|\exp \left(i F\left(s, s_{1} \cdot\right)\right)\right\|_{k} \leq C_{k}\left(1+\left|F\left(s, s_{1}, \cdot\right)\right|_{\infty}\right)^{k-1}\left\|F\left(s, s_{1}, \cdot\right)\right\|_{k}, \quad k \in \mathbb{N} .
$$

For any $s \in J=\left[0, T_{*} \wedge \tau_{2}\right)$, by (A.3) and the definition of $\tau_{2}$, we have that $v:=v(s)$ satisfies

$$
\left\||v|^{2}\right\|_{1} \leq C|v|_{\infty}\|v\|_{1} \leq C|v|_{\infty}\|v\|_{0}^{1-1 / m}\|v\|_{m}^{1 / m} \leq C^{\prime}|v|_{\infty}^{2-1 / m} \nu^{-\kappa}
$$

(this assertion is empty if $\tau_{2}=0$ since then $J=\varnothing$ ). So for $s, s_{1} \in J$,

$\left|F\left(s, s_{1}, \cdot\right)\right|_{\infty} \leqslant\left|s_{1}-s\right| \sup _{s^{\prime} \in J}\left|v\left(s^{\prime}\right)\right|_{\infty}^{2}, \quad\left\|F\left(s, s_{1}, \cdot\right)\right\|_{k} \leq C \nu^{-\kappa k}\left|s_{1}-s\right|\left(\sup _{s^{\prime} \in J}\left|v\left(s^{\prime}\right)\right|_{\infty}\right)^{2-k / m}$ for $k \leq m$. Then, due to (4.16),

$$
\left\|W\left(0, s \wedge \tau_{2}, \cdot\right)\right\|_{1} \leqslant C^{\prime} T_{\star} \nu^{-\kappa}\left(1+\sup _{s \in J}|v(s)|_{\infty}^{2}\right) .
$$

Consider the stochastic integral in (4.15),

$$
N(t, x)=\int_{0}^{t} W(0, s, x) d \xi(s, x) .
$$

The process $t \mapsto W(0, t, x)$ is adapted to the filtration $\left\{\mathcal{F}_{t}\right\}$, and

$$
d W(0, t, x)=i|v(t, x)|^{2} W(0, t, x) d t .
$$

So integrating by parts (see, e.g., [14, Proposition IV.3.1]) we re-write $N$ as

$$
N(t, x)=W(0, t, x) \xi(t, x)-i \int_{0}^{t} \xi(s, x)|v(s, x)|^{2} W(0, s, x) d s,
$$

and we see from (4.15) that

$$
I_{3}(t)=\sqrt{\nu} \xi\left(t \wedge \tau_{2}, x\right)+i \sqrt{\nu} \int_{0}^{t \wedge \tau_{2}} \xi(s, x)|v(s, x)|^{2} W\left(s, t \wedge \tau_{2}, x\right) d s .
$$

Due to (1.4) and since $B_{m}<\infty$, the Wiener process $\xi(t, x)$ satisfies

$$
\mathbb{E}\left\|\xi\left(T_{*}, x\right)\right\|_{1}^{2} \leqslant C B_{1} T_{*},
$$

and

$$
\mathbb{E} \sup _{0 \leqslant t \leqslant T_{*}}|\xi(t, \cdot)|_{\infty} \leqslant \sum_{d \in \mathbb{N}^{n}} b_{d}\left(\mathbb{E} \sup _{0 \leqslant t \leqslant T_{*}}\left|\beta_{d}(t) \varphi_{d}\right|_{\infty}\right) \leqslant C B_{*} \sqrt{T_{*}},
$$

(we recall that $B_{*}=\sum_{d \in \mathbb{N}^{n}}\left|b_{d}\right|<\infty$ ). Therefore,

$$
\mathbb{P}\left(Q_{4}\right) \geqslant 1-\gamma, \quad Q_{4}=\left\{\sup _{0 \leqslant t \leqslant T_{*}}\left(\|\xi(t)\|_{1} \vee|\xi(t)|_{\infty}\right) \leqslant C T_{*}^{1 / 2}\right\},
$$

with a suitable $C=C(\gamma)$. Let

$$
\tilde{Q}=\bigcap_{i=1}^{4} Q_{i},
$$


then $\mathbb{P}(\tilde{Q}) \geqslant \gamma$. As $\tau_{2}=T_{*}$ for $\omega \in \tilde{Q}$, then due to (4.17), (4.18), (4.19) and (A.3), for $\omega \in \tilde{Q}$ we have

$$
\begin{aligned}
\sup _{0 \leqslant t \leqslant T_{*}}\left\|I_{3}^{\omega}(t)\right\|_{1} & \leq \sqrt{\nu} \sup _{0 \leqslant t \leqslant T_{*}}\left(\left\|\xi^{\omega}(t)\right\|_{1}+\int_{0}^{t}\left\|\xi^{\omega}(s)\left|v^{\omega}(s)\right|^{2} W^{\omega}(s, t)\right\|_{1} d s\right) \\
& \leqslant C T_{*}^{5 / 2} \nu^{\frac{1}{2}-\kappa} .
\end{aligned}
$$

Setp 4: We then consider the term $I_{2}=\nu \int_{0}^{t \wedge \tau_{2}} \bar{W}\left(s, t \wedge \tau_{2}, x\right) \Delta v(s, x) d s$. To bound its $H^{1}$-norm we need to estimate $\|W \Delta v\|_{1}$. Since

$$
\left\|\partial_{x}^{a} W \partial_{x}^{b} v\right\|_{0} \leqslant C\|W\|_{3}^{1 / 3}\|v\|_{3}^{2 / 3}|v|_{\infty}^{1 / 3} \quad \text { if }|a|=1,|b|=2,
$$

(see [17, Proposition 3.6]), we have

$$
\|W \Delta v\|_{1} \leqslant C\left(\|v\|_{3}+\|W\|_{3}^{1 / 3}\|v\|_{3}^{2 / 3}|v|_{\infty}^{1 / 3}\right)
$$

Then in view of (4.16) and (4.8), for $\omega \in \tilde{Q}$

$$
\|W \Delta v\|_{1} \leq C\left(\nu^{-3 \kappa}+\left(T_{*}^{3} \nu^{-3 \kappa}\right)^{1 / 3} \nu^{-2 \kappa}\right) \leq C \nu^{-3 \kappa} T_{*},
$$

and accordingly

$$
\sup _{0 \leqslant t \leqslant T_{*}}\left\|I_{2}^{\omega}(t)\right\|_{1} \leqslant \nu \sup _{0 \leqslant t \leqslant T_{*}} \int_{0}^{t}\left\|W^{\omega}\left(s, T_{*}\right) \Delta v^{\omega}(s)\right\|_{1} d s \leqslant C \nu^{1-3 \kappa} T_{*}^{2}, \quad \forall \omega \in \tilde{Q} .
$$

Step 5: $\quad$ Now we estimate from below the $H^{1}$-norm of the term $I_{1}^{\omega}\left(T_{*}, x\right), \omega \in \tilde{Q}$. Writing it as $I_{1}^{\omega}\left(T_{*}, x\right)=e^{-i T_{*}\left|v_{0}(x)\right|^{2}} e^{-i \int_{0}^{T_{*}} \mathscr{E}(s, x) d s} v_{0}(x)$ wee see that

$$
\left\|I_{1}^{\omega}\left(T_{*}\right)\right\|_{1} \geqslant \| \nabla\left(\exp \left(-i T_{*}\left|v_{0}\right|^{2}\right) v_{0}\left\|_{0}-\right\| \nabla\left(\exp \left(-i \int_{0}^{T_{*}} \mathscr{E}(s) d s\right)\right) v_{0}\left\|_{0}-\right\| v_{0} \|_{1} .\right.
$$

This first term on the r.h.s is

$$
T_{*}\left\|v_{0} \nabla\left(\left|v_{0}\right|^{2}\right)\right\|_{0}=T_{*} \frac{2}{3}\left\|\nabla\left|v_{0}\right|^{3}\right\|_{0} \geqslant C T_{*}\left\|\left|v_{0}\right|^{3}\right\|_{0} \geqslant C T_{*}\left\|v_{0}\right\|_{0}^{3} \geq C T_{*} \nu^{6 \kappa}, \quad C>0,
$$

where we have used the fact that $\left.u\right|_{\partial K^{n}}=0$, Poincaré's inequality and (4.7).

For $\omega \in \tilde{Q}$ and $0 \leq s \leq T_{*}$, in view of (4.11), the second term is bounded by

$$
\left\|\left(\int_{0}^{T_{*}} \nabla \mathscr{E}(s) d s\right) v_{0}\right\|_{0} \leqslant C T_{*}\left|v_{0}\right|_{\infty} \sup _{0 \leqslant s \leqslant T_{*}}\|\mathscr{E}(s)\|_{1} \leq C T_{*}\left(\nu^{1-3 \kappa} T_{*}+\nu^{\frac{1}{2}-\kappa} T_{*}^{1 / 2}\right) .
$$

Therefore, using (4.11), we get for the term $I_{1}^{\omega}\left(T_{*}\right)$ the following lower bound:

$$
\left\|I_{1}^{\omega}\left(T_{*}\right)\right\|_{1} \geqslant C\left(\nu^{6 \kappa} T_{*}-T_{*}\left(\nu^{1-3 \kappa} T_{*}+\nu^{\frac{1}{2}-\kappa} T_{*}^{1 / 2}\right)-\nu^{-\kappa}\right) .
$$

Recalling $T_{*}=\nu^{-b}$ we see that if we assume that

$$
\left\{\begin{array}{l}
6 \kappa-b<-\kappa, \\
6 \kappa-b<1-3 \kappa-2 b, \\
6 \kappa-b<1 / 2-\kappa-\frac{3}{2} b,
\end{array}\right.
$$

then for $\omega \in \tilde{Q}$,

$$
\left\|I_{1}^{\omega}\left(T_{*}\right)\right\|_{1} \geqslant C \nu^{6 \kappa} T_{*}, \quad C>0,
$$

provided that $\nu$ is sufficiently small.

Step 6: Finally, remembering that $\tau_{2}=T_{*}$ for $\omega \in \tilde{Q}$ and combining the relations (4.20), (4.21) and (4.23) to estimate the terms of (4.13), we see that for $\omega \in \tilde{Q}$ we have

$$
\left\|v^{\omega}\left(T_{*}\right)\right\|_{1} \geqslant\left\|I_{1}^{\omega}\left(T_{*}\right)\right\|_{1}-\left\|I_{2}^{\omega}\left(T_{*}\right)\right\|_{1}-\left\|I_{3}^{\omega}\left(\tau_{*}\right)\right\|_{1} \geqslant \frac{1}{2} C_{1} \nu^{6 \kappa-b}, \quad C_{1}>0,
$$

if we assume in addition to (4.22) that

$$
6 \kappa-b<\frac{1}{2}-\kappa-\frac{5}{2} b
$$


and $\nu$ is small. Note that this relation implies the last two in (4.22).

Combining (4.8) and (4.24) we get that

$$
\nu^{-b+7 \kappa} \leqslant C_{2}^{-1}
$$

for all sufficiently small $\nu$. Thus we have obtained a contradiction with the existence of the sets $Q^{\nu_{j}}$ as at the beginning of the proof if (for a chosen $\kappa$ ) we can find a $b \in(0,1)$ which meets $(4.22),(4.25)$ and

$$
-b+7 \kappa<0 .
$$

Noting that this is nothing but the first relation in (4.22), we see that we have obtained a contradiction if

$$
\kappa<\frac{1}{7} b, \quad \kappa<\frac{1}{14}-\frac{3}{14} b,
$$

for some $b \in(0,1)$. We see immediately that such a $b$ exists if and only if $\kappa<\frac{1}{35}$.

Amplification. If we replace the condition $m \geqslant 3$ with the weaker assumption

$$
\mathbb{R} \ni m>2 \text {, }
$$

then the statement (4.2) remains true for $0<\kappa<\kappa(n, m)$ with a suitable (less explicit) constant $\kappa(n, m)>0$. In this case we obtain a contradiction with the assumption (4.3) by deriving a lower bound for $\left\|v\left(T_{*}\right)\right\|_{\alpha}$, where $\alpha=\min \{1, m-2\} \epsilon$ $(0,1]$, using the decomposition (4.13). The proof remains almost identical except that now, firstly, we bound $\left\|I_{2}\right\|_{\alpha}(\alpha<1)$ from above using the following estimate from [15, Theorem 5, p. 206] (also see there p. 14):

$$
\|W \Delta u\|_{\alpha} \leqslant C\|u\|_{2+\alpha}\left(|W|_{\infty}+|W|_{\infty}^{1-\frac{2 \alpha}{n}}\|W\|_{2}^{\frac{2 \alpha}{n}}\right) ;
$$

and, secondly, estimate $\left\|I_{1}^{\omega}\left(T_{*}\right)\right\|_{\alpha}(\alpha<1)$ from below as

$$
\left\|I_{1}^{\omega}\left(T_{*}\right)\right\|_{\alpha} \geqslant\left\|I_{1}^{\omega}\left(T_{*}\right)\right\|_{1}^{2-\alpha}\left\|I_{2}^{\omega}\left(T_{*}\right)\right\|_{2}^{-1+\alpha},
$$

which directly follows from Sobolev's interpolation. See [6] for more details.

\section{LOWER BOUNDS FOR TIME-AVERAGED SOBOLEV NORMS}

In this section we prove the assertion (2) of Theorem 1. We provide each space $H^{r}, r \geq 0$, with the scalar product

$$
\left.\langle u, v\rangle_{r}:=\left\langle(-\Delta)^{\frac{r}{2}} u,(-\Delta)^{\frac{r}{2}} v\right\rangle\right\rangle,
$$

corresponding to the norm $\|u\|_{r}$. Let $u(t)=\sum u_{d}(t) \varphi_{d}$ be a solution of eq. (1.1). Applying Itô's formula to the functional $\|u\|_{m}^{2}$, we have for any $0 \leqslant t<t^{\prime}<\infty$ the relation

$$
\begin{aligned}
\left\|u\left(t^{\prime}\right)\right\|_{m}^{2}= & \|u(t)\|_{m}^{2}+2 \int_{t}^{t^{\prime}}\left\langle u(s), \nu \Delta u(s)-i|u(s)|^{2} u(s)\right\rangle_{m} d s \\
& +2 \nu B_{m}\left(t^{\prime}-t\right)+2 \sqrt{\nu} M\left(t, t^{\prime}\right),
\end{aligned}
$$

where $M$ is the stochastic integral

$$
M\left(t, t^{\prime}\right):=\int_{t}^{t^{\prime}} \sum_{d \in \mathbb{N}^{n}} b_{d}|d|^{2 m}\left\langle u_{d}(s), d \beta_{d}(s)\right\rangle .
$$

Let us fix a $\gamma \in\left(0, \frac{1}{8}\right)$. Due to Theorems 7 and 13, for small enough $\nu$ there exists an event $\Omega_{1} \subset \Omega, \mathbb{P}\left(\Omega_{1}\right) \geqslant 1-\gamma / 2$, such that for all $\omega \in \Omega_{1}$ we have:

a) $\sup _{0 \leqslant t \leqslant \frac{1}{\nu}}\left|u^{\omega}(t)\right|_{\infty} \leqslant C(\gamma)$, for a suitable $C(\gamma)>0$;

b) there exist $t_{\omega} \in\left[0, \frac{1}{3 \nu}\right]$ and $t_{\omega}^{\prime} \in\left[\frac{2}{3 \nu}, \frac{1}{\nu}\right]$ satisfying

$$
\left\|u^{\omega}\left(t_{\omega}\right)\right\|_{m},\left\|u^{\omega}\left(t_{\omega}^{\prime}\right)\right\|_{m} \geqslant \nu^{-m \kappa} .
$$


Since for the martingale $M(0, t)$ we have that

$$
\mathbb{E}\left|M\left(0, \frac{1}{\nu}\right)\right|^{2} \leqslant B_{m} \mathbb{E} \int_{0}^{\frac{1}{\nu}}\|u(s)\|_{m}^{2} d s=: X_{m},
$$

then by Doob's inequality

$$
\mathbb{P}\left(\Omega_{2}\right) \geqslant 1-\frac{\gamma}{2}, \quad \Omega_{2}=\left\{\sup _{0 \leqslant t \leqslant \frac{1}{\nu}}|M(0, t)| \leqslant c(\gamma) X_{m}^{1 / 2}\right\} .
$$

Now let us set $\hat{\Omega}=\Omega_{1} \cap \Omega_{2}$. Then $\mathbb{P}(\hat{\Omega}) \geqslant 1-\gamma$ for small enough $\nu$, and for any $\omega \in \hat{\Omega}$ there are two alternatives:

i) there exists a $t_{\omega}^{0} \in\left[0, \frac{1}{3 \nu}\right]$ such that $\left\|u^{\omega}\left(t_{\omega}^{0}\right)\right\|_{m}=\frac{1}{3} \nu^{-\kappa m}$. Then from (5.1) and (5.2) in view of (A.4) we get

$\frac{8}{9} \nu^{-2 m \kappa}+2 \nu \int_{t_{\omega}^{0}}^{t_{\omega}^{\prime}}\left\|u^{\omega}(s)\right\|_{m+1}^{2} d s \leqslant C(m, \gamma) \int_{0}^{\frac{1}{\nu}}\left\|u^{\omega}(s)\right\|_{m}^{2} d s+2 B_{m}+2 \sqrt{\nu} c(\gamma) X_{m}^{1 / 2}$.

ii) There exists no $t \in\left[0, \frac{1}{3 \nu}\right]$ with $\left\|u^{\omega}(t)\right\|_{m}=\frac{1}{3} \nu^{-\kappa m}$. In this case, since $\left\|u^{\omega}(t)\right\|_{m}$ is continuous with respect to $t$, then due to (5.2) $\left\|u^{\omega}(t)\right\|_{m}>\frac{1}{3} \nu^{-m \kappa}$ for all $t \in\left[0, \frac{1}{3 \nu}\right]$. This leads to the relation

$$
\frac{1}{27} \nu^{-2 m \kappa-1} \leqslant \int_{0}^{\frac{1}{\nu}}\left\|u^{\omega}(s)\right\|_{m}^{2} d s .
$$

In both cases for $\omega \in \hat{\Omega}$ we have:

It implies that

$$
\frac{1}{27} \nu^{-2 m \kappa} \leqslant C^{\prime}(m, \gamma) \int_{0}^{\frac{1}{\nu}}\|u(s)\|_{m}^{2} d s+2 B_{m}+\nu c(\gamma)^{2}+X_{m}
$$

$$
\mathbb{E} \nu \int_{0}^{\frac{1}{\nu}}\|u(\tau)\|_{m}^{2} d \tau \geqslant C \nu^{-2 m \kappa+1}
$$

(for small enough $\nu$ ), and gives the lower bound in (1.7).

The upper bound follows directly from Theorem 8.

Proof of Corollaries 3 and 4: Since $B_{k}<\infty$ for each $k$ and all coefficients $b_{d}$ are non-zero, then eq. (1.1) is mixing in the spaces $H^{m}, m \in \mathbb{N}$, see Corollary 10. As the stationary solution $v^{s t}$ satisfies Corollary 11 with any $m$, then for each $\mu \in \mathbb{N}$ and $M>0$, interpolating the norm $\|u\|_{\mu}$ via $\|u\|_{0}$ and $\|u\|_{m}$ with $m$ sufficiently large we get that the stationary measure $\mu_{\nu}$ satisfies

$$
\int\|u\|_{\mu}^{M} \mu_{\nu}(d u)<\infty \quad \forall \mu \in \mathbb{N}, \forall M>0 .
$$

Similar, in view of (2.5) and Theorem 7 ,

$$
\mathbb{E}\left\|u\left(t ; u_{0}\right)\right\|_{\mu}^{M} \leq C_{\nu}\left(u_{0}\right) \quad \forall t \geq 0
$$

for each $u_{0} \in C^{\infty}$ and every $\mu$ and $M$ as in (5.3). Now let us consider the integral in (1.7) and write it as

$$
J_{t}:=\nu \int_{t}^{t+\nu^{-1}} \mathbb{E}\|u(s)\|_{m}^{2} d s .
$$

Replacing the integrand in $J_{t}$ with $\mathbb{E}\left(\left\|u_{\nu}(s)\right\|_{m} \wedge N\right)^{2}, N \geq 1$, using the convergence

$$
\mathbb{E}\left(\left\|u\left(s ; v_{0}\right)\right\|_{m} \wedge N\right)^{2} \rightarrow \int\left(\|u\|_{m} \wedge N\right)^{2} \mu_{\nu}(d u) \quad \text { as } \quad s \rightarrow \infty \quad \forall N
$$

which follows from Corollary 10, and the estimates (5.3), (5.4) we get that

$$
J_{t} \rightarrow \int\|u\|_{m}^{2} \mu_{\nu}(d u) \quad \text { as } \quad t \rightarrow \infty
$$

This convergence and (1.7) imply the assertion of Corollary 3.

Now the convergence (5.5) jointly with estimates (5.3), (5.4) and (1.8) imply Corollary 4. 


\section{Appendix A. Some estimates}

For any integer $l \in \mathbb{N}$ and $F \in H^{l}$ we have that

$$
\|\exp (i F(x))\|_{l} \leq C_{l}\left(1+|F|_{\infty}\right)^{l-1}\|F\|_{l} .
$$

Indeed, to verify (A.1) it suffices to check that for any non-zero multi-indices $\beta_{1}, \ldots, \beta_{l^{\prime}}$, where $1 \leq l^{\prime} \leqslant l$ and $\left|\beta_{1}\right|+\cdots+\left|\beta_{l^{\prime}}\right|=l$, we have

$$
\left\|\partial_{x}^{\beta_{1}} F \cdots \partial_{x}^{\beta_{x^{\prime}}} F\right\|_{0} \leq C|F|_{\infty}^{l^{\prime}-1}\|F\|_{l} .
$$

But this is the assertion of Lemma 3.10 in [17]. Similarly,

$$
\|F G\|_{r} \leqslant C_{r}\left(|F|_{\infty}\|G\|_{r}+|G|_{\infty}\|F\|_{r}\right), \quad F, G \in H^{r}, \quad r \in \mathbb{N},
$$

see [17, Proposition 3.7] (this relation is known as Moser's estimate). Finally, since for $|\beta| \leq m$ we have $\left|\partial_{x}^{\beta} v\right|_{2 m / \beta \mid} \leq C|v|_{\infty}^{1-|\beta| / m}\|v\|_{m}^{|\beta| / m}$ (see relation (3.17) in [17]), then

$$
\left|\left\langle|v|^{2} v, v\right\rangle_{m}\right| \leq C_{m}\|v\|_{m}^{2}|v|_{\infty}^{2}, \quad\left|\left\langle|v|^{2} v, v\right\rangle_{m}\right| \leq C_{m}^{\prime}\|v\|_{m+1}^{\frac{2 m}{m+1}}|v|_{\infty}^{\frac{2 m+4}{m+1}} .
$$

\section{Appendix B. Proof of Theorem 8}

Applying Ito's formula to a solution $v(\tau)$ of eq. (2.1) we get a slow time version of the relation (5.1):

$$
\|v(\tau)\|_{m}^{2}=\left\|v_{0}\right\|_{m}^{2}+2 \int_{0}^{\tau}\left(-\|v\|_{m+1}^{2}-\nu^{-1}\left\langle i|v|^{2} v, v\right\rangle_{m}\right) d s+2 B_{m} \tau+2 M(\tau),
$$

where $M(\tau)=\int_{0}^{\tau} \sum_{d} b_{d}|d|^{2 m}\left\langle v_{d}(s), d \beta_{d}(s)\right\rangle$. Since in view of (A.4)

$$
\mathbb{E}\left|\left\langle|v|^{2} v, v\right\rangle_{m}\right| \leq C_{m}\left(\mathbb{E}\|v\|_{m+1}^{2}\right)^{\frac{m}{m+1}} \mathbb{E}\left(|v|_{\infty}^{2 m+4}\right)^{\frac{1}{m+1}},
$$

then denoting $\mathbb{E}\|v(\tau)\|_{r}^{2}=: g_{r}(\tau), r \in \mathbb{N} \cup\{0\}$, taking the expectation of (B.1), differentiating the result and using (2.3), we get that

$$
\frac{d}{d \tau} g_{m} \leq-2 g_{m+1}+C_{m} \nu^{-1} g_{m+1}^{\frac{m}{m+1}}+2 B_{m} \leq-2 g_{m+1}\left(1-C_{m}^{\prime} \nu^{-1} g_{m}^{-\frac{1}{m}}+2 B_{m}\right),
$$

since $g_{m} \leq g_{0}^{1 /(m+1)} g_{m+1}^{m /(m+1)} \leq C_{m} g_{m+1}^{m /(m+1)}$. We see that if $g_{m} \geq\left(2 \nu^{-1} C_{m}^{\prime}\right)^{m}$, then the r.h.s. of (B.2) is

$$
\leq-g_{m+1}+2 B_{m} \leq-C_{m}^{-1} g_{m}^{(m+1) / m}+2 B_{m} \leq-\bar{C}_{m} \nu^{-m-1}+2 B_{m},
$$

which is negative if $\nu \ll 1$. So if

$$
g_{m}(\tau)<\left(2 \nu^{-1} C_{m}^{\prime}\right)^{m}
$$

at $\tau=0$, then (B.4) holds for all $\tau \geq 0$ and (2.4) follows. If $g_{m}(0)$ violates (B.4), then in view of (B.2) and (B.3), for $\tau \geq 0$, while (B.4) is false, we have that

$$
\frac{d}{d \tau} g_{m} \leq-C_{m} g_{m}^{(m+1) / m}+2 B_{m}
$$

which again implies (2.4).Besides, in view of (B.2),

$$
\frac{d}{d \tau} g_{m} \leq-g_{m}+C_{m}\left(\nu,\left|v_{0}\right|_{\infty}, B_{m_{*}}, B_{m}\right) .
$$

This relation immediately implies (2.5).

Now let us return to eq. (B.1). Using Doob's inequality and (2.4) we find that

$$
\mathbb{E}\left(\sup _{0 \leq \tau \leq T}|M(\tau)|^{2}\right) \leq C<\infty .
$$

Next, applying (A.4) and Young's inequality we get

$$
\int_{0}^{\tau}\left(-\|v\|_{m+1}^{2}-\nu^{-1}\left\langle i|v|^{2} v, v\right\rangle_{m}\right) d s \leq C_{m} \int_{0}^{\tau}|v(s)|_{\infty}^{2 m+3} d s, \quad \forall 0 \leq \tau \leq T .
$$


Finally, using in (B.1) the last two displayed formulas jointly with (2.3) we obtain $(2.6)$.

\section{ACKNOWLEDGMENT}

GH is supported by NSFC (Significant project No.11790273) in China and SK thanks the Russian Science Foundation for support through the grant 18-11-00032.

\section{REFERENCES}

[1] M. Bartuccelli, J. Gibbon, and M. Oliver. Length scales in solutions of the complex GinzburgLandau equation. Physica D, 89:267-286, 1996.

[2] A. Biryuk. Lower bounds for derivatives of solutions for nonlinear Schrödinger equations. Proceedings A of the Royal Society of Edinburgh, 139:237-251, 2009.

[3] T. Bohr, M. Jensen, G. Paladin, and A. Vulpiani. Dynamical Systems Approach to Turbulence. Cambridge University Press, 1998.

[4] J. Colliander, M. Keel, G. Staffilani, H. Takaoka, and T. Tao. Transfer of energy to high frequencies in the cubic defocusing nonlinear Schrödinger equation. Inv. Math., 181:39-113, 2010.

[5] U. Frisch. Turbulence: the Legacy of A. N. Kolmogorov. Cambridge University Press, Cambridge, 1995.

[6] G. Huang and S. Kuksin. On the energy transfer to high frequencies in damped/driven nonlinear Schrödinger equation (extended version). arXiv 2006.11518, 2020.

[7] N. V. Krylov. Controlled Diffusion Processes. Springer, 1980.

[8] S. Kuksin. A stochastic nonlinear Schrödinger equation. I. A priori estimates. Tr. Mat. Inst. Steklov, 225:232-256, 1999.

[9] S. Kuksin. Spectral properties of solutions for nonlinear PDEs in the turbulent regime. GAFA, 9:141-184, 1999.

[10] S. Kuksin and V. Nersesyan. Stochastic CGL equations without linear dispersion in any space dimension. Stoch PDE: Anal. Comp., 1(3):389-423, 2013.

[11] S. Kuksin and A. Shirikyan. Mathematics of Two-Dimensional Turbulence. Cambridge University Press, 2012.

[12] S. B. Kuksin and A. Shirikyan. Randomly forced CGL equation: stationary measures and the inviscid limit. J. Phys. A: Math. Gen., 37:1-18, 2004.

[13] C.D. Levermore and M. Oliver. The complex Ginzburg-Landau equation as a model problem. Lectures in Applied Math., 31:141-189, 1996.

[14] D. Revuz and M. Yor. Continuous martingales and Brownian Motion. Springer, 2005.

[15] T. Runst and W. Sickel. Sobolev Spaces of Fractional Order, Nemytskij Operators, and Nonlinear Partial Differential Equations. Walter de Gruyter, Berlin and New York, 2011.

[16] A. Shirikyan. Local times for solutions of the complex Ginzburg-Landau equation and the inviscid limit. J. Math. Anal. Appl., 384:130-137, 2011.

[17] M. E. Taylor. Partial Differential Equations III. Applied Mathematical Sciences. Springer, 2011.

Yau Mathematical Sciences Center, Tsinghua University, Beijing, China

E-mail address: huangguan@tsinghua.edu.cn

Institut de Mathémathiques de Jussieu-Paris Rive Gauche, CNRS, Université Paris Diderot, UMr 7586, Sorbonne Paris Cité, F-75013, Paris, France \& School of Mathematics, Shandong University, Jinan, Shandong, China \& Saint Petersburg State University, Universitetskaya nab., St. Petersburg, Russia

E-mail address: Sergei.Kuksin@imj-prg.fr 